\title{
Variational Iteration Method for Solving the Generalized Degasperis-Procesi Equation
}

\author{
Qian Lijuan, ${ }^{1}$ Tian Lixin, ${ }^{2}$ and Ma Kaiping ${ }^{3}$ \\ ${ }^{1}$ Faculty of Science, Jiangsu University, Zhenjiang, Jiangsu Province 212013, China \\ ${ }^{2}$ Nanjing Normal University, Nanjing, Jiangsu Province 210097, China \\ ${ }^{3}$ College of Engineering, Nanjing Agricultural University, Nanjing, Jiangsu Province 210031, China
}

Correspondence should be addressed to Qian Lijuan; qianlj2013@hotmail.com

Received 31 December 2013; Accepted 10 February 2014; Published 13 March 2014

Academic Editor: Baojian Hong

Copyright (C) 2014 Qian Lijuan et al. This is an open access article distributed under the Creative Commons Attribution License, which permits unrestricted use, distribution, and reproduction in any medium, provided the original work is properly cited.

\begin{abstract}
We introduce the variational iteration method for solving the generalized Degasperis-Procesi equation. Firstly, according to the variational iteration, the Lagrange multiplier is found after making the correction functional. Furthermore, several approximations of $u_{n+1}(x, t)$ which is converged to $u(x, t)$ are obtained, and the exact solutions of Degasperis-Procesi equation will be obtained by using the traditional variational iteration method with a suitable initial approximation $u_{0}(x, t)$. Finally, after giving the perturbation item, the approximate solution for original equation will be expressed specifically.
\end{abstract}

\section{Introduction}

The theory of soliton has extensive applications in physics, mechanics, and combustion science. In recent years, many researchers studied the soliton theory in the fields of shock wave [1,2], light scattering, quantum mechanics, atmospheric physics, neural networks, explosion, and combustion [3]. There are many new methods for searching the soliton solution of nonlinear evolution equations such as hyperbolic tangent function method [4], the homogeneous balance methods [5], Jacobi elliptic function expansion method [3], and pseudo-spectral method [6].

The variational iteration method (VIM) was developed, in 1999, by He [7-13]. The VIM gives rapidly convergent successive approximations of the exact solution if such a solution exists; otherwise, a few approximations can be used for numerical purposes. The Adomian decomposition method suffers from the complicated computational work needed for the derivation of Adomian polynomials for nonlinear terms. The VIM has no specific requirements, such as linearization, small parameters for nonlinear operators. Therefore, the VIM can overcome the foregoing restrictions and limitations of perturbation techniques, so that it provides us with a possibility to analyze strongly nonlinear problems.
On the other hand, the VIM is capable of greatly reducing the size of calculation while still maintaining high accuracy of the numerical solution [14]. Moreover, the power of the method gives it a wider applicability in handling a huge number of analytical and numerical applications. The VIM was successfully applied to study a variety of differential equations. It is based on Lagrange multiplier, and it has the merits of simplicity and easy execution. As a result, it has been proved by many authors to be a powerful mathematical tool for addressing various kinds of linear and nonlinear problem. For example, this method was used for solving nonlinear wave equations and the Laplace equation by Wazwaz [14]. The VIM for solving linear systems of ODEs with constant coefficients was studied by Khojasteh Salkuyeh [15]. Helmholtz equation was researched by Momani and Abuasad [16]. Geng [17] introduced the piecewise VIM for solving Riccati differential equation. Fractional vibration equation was researched by Das [18]. Furthermore, higher order boundary value problems were researched by $\mathrm{Xu}$ [19],Noor, and Mohyud-Din [20]. Noor et al. [21] applied a modified He's variational iteration method for solving singular fourthorder parabolic partial differential equations. The proposed modification is made by introducing He's polynomials in the correction functional. Ghorbani and Saberi-Nadjafi [22] 
modified the VIM by constructing an initial trial function without unknown parameters. Sevimlican [23] constructed approximate Green's function for a vector equation for the electric field by using VIM.

In this paper, we are concerned with the variational iterations method for solving the generalized DegasperisProcesi equation. As a review, we will recall the VIM briefly in Section 2.

\section{Variational Iteration Method}

In this section, the basic concepts of variational iteration method (VIM) are introduced. Here, a description of method [7-15] is given to handle the general nonlinear problem. Consider the differential equation of the form

$$
L u(x, t)+N u(x, t)=f(x, t),
$$

where $L$ is a linear operator, $N$ is a nonlinear operator, and $f(x, t)$ is the inhomogeneous term. According to He's variational iteration method, we can construct a correction functional for (1) as follows:

$$
\begin{aligned}
u_{n+1}(x, t) & \\
= & u_{n}(x, t) \\
& \quad+\int_{0}^{t} \lambda(\tau)\left(L u_{n}(x, \tau)+N \tilde{u}_{n}(x, \tau)-f(x, \tau)\right) d \tau,
\end{aligned}
$$

where $\lambda$ is a general Lagrange multiplier, which can be identified optimally via variational theory $[12,24]$. Here $\tilde{u}_{n}$ is considered as a restricted variation $[14,25]$ which means $\delta \tilde{u}_{n}=0$; the subscript $n$ denotes the $n$th approximations. The successive approximations $u_{n+1}(x, t)$, of the solution $u(x, t)$, can be obtained after using the obtained Lagrange multiplier and the zeroth approximation $u_{0}(x, t)$, which are selected from any function that satisfies the initial conditions. With $\lambda$ determined, several approximations $u_{n+1}(x, t), n \geqslant 0$ follow. Consequently, the exaction solution may be obtained as

$$
u(x, t)=\lim _{n \rightarrow+\infty} u_{n+1}(x, t) .
$$

In fact, the VIM depends on the suitable selection of the initial approximation $u_{0}(x, t)$. Moreover, we use a well-known, powerful tool to prove the convergence of the sequence obtained via the VIM and its rate. It is the Banach's fixed point theorem that follows.

Theorem 1 (Banach's fixed point theorem). Assume that $X$ is a Banach space and

$$
A: X \longrightarrow X
$$

is a nonlinear mapping, and suppose that

$$
\|A[u]-A[v]\| \leqslant \alpha\|u-v\|, \quad u, v \in X
$$

for some constant $\alpha<1$. Then $A$ has a unique fixed point. Furthermore, the sequence

$$
u_{n+1}=A\left[u_{n}\right]
$$

with an arbitrary choice of $u_{0} \in X$ converges to the fixed point of $A$.

According to Theorem 1, for the nonlinear mapping

$$
\begin{aligned}
& A\left[u_{n}(x, t)\right] \\
& \quad=u_{n}(x, t) \\
& \quad+\int_{0}^{t} \lambda(\tau)\left\{L u_{n}(x, \tau)+N(x, \tau)-f(x, \tau)\right\} d \tau
\end{aligned}
$$

a sufficient condition for the convergence of the variational iteration method is strict contraction of $A$. Furthermore, the sequence (2) converges to the fixed point of $A$ which is also the solution of problem (1). Some modifications to prove the convergence speed and to lengthen the interval of convergence for VIM series solution are suggested in $[17,26-$ 30].

\section{The Variational Iteration of Generalized Degasperis-Procesi Equation}

Degasperis and Procesi consider the following family of third-order dispersive conservation laws [31],

$$
u_{t}+c_{0} u_{x}+\gamma u_{x x x}-\alpha^{2} u_{x x t}=\left(c_{1} u^{2}+c_{2} u_{x}^{2}+c_{3} u u_{x x}\right)_{x}
$$

where $\alpha, \gamma, c_{0}, c_{1}, c_{2}$, and $c_{3}$ are real constants. In this family, only three equations satisfy asymptotic integrability conditions [31]. That is, if $c_{0}=1, c_{1}=-1 / 2, c_{2}=0, c_{3}=0$, $\alpha^{2}=0$, and $\gamma=1,(8)$ is the $\mathrm{KdV}$ equation

$$
u_{t}+u_{x}+u u_{x}+u_{x x x}=0
$$

If $c_{0}=0, c_{1}=-3 / 2, c_{2}=1 / 2, c_{3}=1, \alpha^{2}=1$, and $\gamma=0$, (8) is the Camassa-Holm equation

$$
u_{t}-u_{x x t}+3 u u_{x}=2 u_{x} u_{x x}+u u_{x x x} .
$$

If $c_{0}=0, c_{1}=-2, c_{2}=1, c_{3}=1, \alpha^{2}=1$, and $\gamma=0$, (8) is the Degasperis-Procesi equation

$$
u_{t}-u_{x x t}+4 u u_{x}=3 u_{x} u_{x x}+u u_{x x x} .
$$

It should be mentioned that both $\mathrm{C}-\mathrm{H}$ and $\mathrm{D}-\mathrm{P}$ equations are derived as members of a one-parameter family of asymptotic shallow water approximations to the Euler equations. It shows that the two equations are physically relevant; otherwise, the $D-P$ equation would be of purely theoretical interest.

Variational iteration method for KdV-Burgers and Lax's seventh-order KdV equations has been studied by Soliman [32]. In this paper, we consider the generalized DegasperisProcesi equation

$$
\begin{aligned}
u_{t}-u_{x x t} & +4 u u_{x}-3 u u_{x x}-u u_{x x x} \\
= & f\left(u, u_{x}, u_{t}, u_{x x}, u_{x x x}, u_{x x t}\right)
\end{aligned}
$$

that was proposed in [33]. $f$ is the generalized perturbation item. We suppose $f$ is a sufficiently smooth function of the variable. 
Step 1. Make the independent variable transformation:

$$
\xi=k(x-\omega t)+\xi_{0}
$$

Here, $\xi_{0} \in C$ is an arbitrary complex number. $k$ is wave number; $\omega$ is wave velocity. Substituting (13) into (12), we have

$$
-\omega u^{\prime}+k^{2} \omega u^{\prime \prime \prime}+4 u u^{\prime}-3 k u u^{\prime \prime}-k^{2} u u^{\prime \prime \prime}=f_{1} .
$$

Here, $u^{\prime}$ is the derivative of $u$ with respect to $\xi$; that is, $u^{\prime}=$ $d u / d \xi . f_{1}=f_{1}\left(u, u^{\prime}, u^{\prime \prime}, u^{\prime \prime \prime}\right)$.

Step 2. From [34], we find the special solution, when $f$ is identical to 0 :

$$
u_{0}(\xi)=-\frac{1}{2}+\frac{1}{2} \tanh ^{2}\left[\frac{1}{2}(x-\omega t)+\xi_{0}\right]
$$

Remark 2. Notice that $\xi_{0} \in C, i \tanh (i \xi)=-\tan \xi, \tanh (\xi+$ $(\pi i / 2))=\operatorname{coth} \xi, i \operatorname{coth}(i \xi)=\cot \xi$, and $\tanh [(1 / 2)(\xi+$ $(i / 2) \pi)]=\tanh \xi+i \operatorname{sech} \xi$, where $i=\sqrt{-1}$. These solutions contain the other four types of forms named $\operatorname{coth} \xi \tan \xi$, $\cot \xi$, and $\tanh \xi+i \operatorname{sech} \xi$.

Step 3. Make the correction functional

$$
u_{n+1}(\xi)
$$

$$
\begin{aligned}
=u_{n}(\xi)-\int_{0}^{\xi} \lambda(s)[ & -\omega \tilde{u}_{n}^{\prime}+k^{2} \omega u_{n}^{\prime \prime \prime}+4 \widetilde{u}_{n} \tilde{u}_{n}^{\prime} \\
& \left.-3 k \widetilde{u}_{n} \widetilde{u}_{n}^{\prime \prime}-k^{2} \widetilde{u}_{n} \tilde{u}_{n}^{\prime \prime \prime}-\tilde{f}_{1}\right] d s .
\end{aligned}
$$

Here, $\widetilde{u}_{n}, \widetilde{u}_{n}^{\prime}, \widetilde{u}_{n}^{\prime \prime}$, and $\widetilde{u}_{n}^{\prime \prime \prime}$ are considered as a restricted variation [35]. That is,

$$
\delta \widetilde{u}_{n}=\delta \widetilde{u}_{n}^{\prime}=\delta \widetilde{u}_{n}^{\prime \prime}=\delta \widetilde{u}_{n}^{\prime \prime \prime}=0 .
$$

Step 4. Under the above condition, make the correct functional stationary with respect to $u_{n}$; noticing that $\delta u_{n}(0)=0$, we have

$$
\begin{aligned}
& \delta u_{n+1}(\xi) \\
& =\delta u_{n}(\xi)-\delta \int_{0}^{\xi} \lambda(s)\left[-\omega \widetilde{u}_{n}^{\prime}+k^{2} \omega u_{n}^{\prime \prime \prime}+4 \widetilde{u}_{n} \tilde{u}_{n}^{\prime}\right. \\
& \left.\quad-3 k^{2} \widetilde{u}_{n} \widetilde{u}_{n}^{\prime \prime}-k^{2} \widetilde{u}_{n} \widetilde{u}_{n}^{\prime \prime \prime}-\tilde{f}\right] d s \\
& =\delta u_{n}(\xi)-\left[\left.\lambda \delta u^{\prime \prime}(s)\right|_{s=\xi}-\left.\lambda^{\prime}(s) \delta u^{\prime}(s)\right|_{s=\xi}\right. \\
& \left.+\left.\lambda^{2}(s) \delta u(s)\right|_{s=\xi}-\int_{0}^{\xi} \lambda^{\prime \prime \prime}(s) \delta u d s\right]=0 .
\end{aligned}
$$

For arbitrary $\delta u_{n+1}$, from the above relation, we obtain the Euler-Language equation:

$$
\begin{gathered}
1-\left.k^{2} \omega \lambda^{\prime \prime}(s)\right|_{s=\xi}=0, \\
\left.k^{2} \omega \lambda^{\prime \prime \prime}(s)\right|_{s=\xi}=0, \\
\left.k^{2} \omega \lambda^{\prime}(s)\right|_{s=\xi}=0, \\
\left.k^{2} \omega \lambda(s)\right|_{s=\xi}=0 .
\end{gathered}
$$

Solve (19), we derive

$$
\lambda(s)=-\frac{1}{2} \cdot \frac{1}{k^{2} \omega}(s-\xi)^{2} .
$$

Substituted (20) into (16), we have the integration form:

$$
\begin{aligned}
& u_{n+1} \\
& =u_{n} \\
& +\int_{0}^{\xi} \frac{1}{2 k^{2} \omega}(s-\xi)^{2} \\
& \quad \times\left[k^{2} \omega u_{n}^{\prime \prime \prime}-\omega u_{n}^{\prime}+4 u_{n} u_{n}^{\prime}\right. \\
& \left.\quad-3 k u_{n} u_{n}^{\prime \prime}-k^{2} u_{n} u_{n}^{\prime \prime \prime}-f_{1}\right] d s, \quad n=0,1,2, \ldots
\end{aligned}
$$

From the above solution procedure, we can see that the approximate solutions converge to its exact solution. That is, $u(x, t)=\lim _{n \rightarrow \infty} u_{n}(x, t), u_{n}(x, t)$ is the approximate solution with arbitrary degree of accurate solitary wave of Degasperis-Procesi equation.

Step 5. Calculation of the approximate solution.

According to the integration form (21),we can calculate the approximate solution. Firstly, let (15) be the zero-order approximate solution:

$$
u_{0}(\xi)=-\frac{1}{2}+\frac{1}{2} \tanh ^{2}\left[\frac{1}{2}(x-\omega t)+\xi_{0}\right] .
$$

Substitute (15) into (21). We obtain the one-order approximate solution $u_{1}(\xi)$

$$
\begin{aligned}
u_{1}(\xi) & \\
= & u_{0}(\xi)+\int_{0}^{\xi} \frac{1}{2 k^{2} \omega}(s-\xi)^{2}\left(-f_{1}\left(u_{0}\right)\right) d s \\
= & -\frac{1}{2}+\frac{1}{2} \tanh ^{2}\left[\frac{1}{2}(x-\omega t)+\xi_{0}\right] \\
& -\int_{0}^{\xi} \frac{1}{2 k^{2} \omega}(s-\xi)^{2} f_{1}\left(u_{0}(s), u_{0}^{\prime}(s), u_{0}^{\prime \prime}(s), u_{0}^{\prime \prime \prime}(s)\right) d s \\
= & -\frac{1}{2}+\frac{1}{2} \tanh ^{2}\left[\frac{1}{2}(x-\omega t)+\xi_{0}\right]+v_{0}(\xi),
\end{aligned}
$$


TABLE 1: Numerical example for the solution of DP equation, $\xi_{0}=0, \omega=1, t=0$, and $\varepsilon=0.1$.

\begin{tabular}{lccc}
\hline$x$ & Initial solution $u_{0}$ & 1th approximate solution $u_{1}$ & Absolute error \\
\hline 0.1 & -0.498752 & -0.498762 & $-4.16770 * 10^{-6}$ \\
0.11 & -0.498491 & -0.498497 & $5.54752 * 10^{-6}$ \\
0.12 & -0.498204 & -0.498211 & $7.2026 * 10^{-6}$ \\
0.13 & -0.497893 & -0.497902 & $9.15804 * 10^{-6}$ \\
0.14 & -0.497558 & -0.497694 & $1.53894 * 10^{-5}$ \\
0.15 & -0.497198 & -0.497212 & $1.407042 * 10^{-5}$ \\
0.16 & -0.496814 & -0.496831 & $1.706776 * 10^{-5}$ \\
0.17 & -0.496405 & -0.496426 & $2.04856 * 10^{-5}$ \\
0.18 & -0.495972 & -0.495996 & $2.43198 * 10^{-5}$ \\
0.19 & -0.495515 & -0.495543 & $2.86050 * 10^{-5}$ \\
0.2 & -0.495033 & -0.495066 & $3.33668 * 10^{-5}$ \\
\hline
\end{tabular}

TABLE 2: Numerical example for the solution of DP equation, $\xi_{0}=0, \omega=1, t=0$, and $\varepsilon=0.05$.

\begin{tabular}{lccc}
\hline$x$ & Initial solution $u_{0}$ & 1th approximate solution $u_{1}$ & Absolute error \\
\hline 0.1 & -0.498752 & -0.4987524 & $2.08385 * 10^{-6}$ \\
0.11 & -0.498491 & -0.498493 & $2.77376 * 10^{-6}$ \\
0.12 & -0.498204 & -0.4982076 & $3.6013 * 10^{-6}$ \\
0.13 & -0.497893 & -0.4978976 & $4.57902 * 10^{-6}$ \\
0.14 & -0.497558 & -0.4975637 & $5.71902 * 10^{-6}$ \\
0.15 & -0.497198 & -0.497205 & $7.03521 * 10^{-6}$ \\
0.16 & -0.496814 & -0.4968225 & $8.5388 * 10^{-6}$ \\
0.17 & -0.496405 & -0.496415 & $1.02428 * 10^{-5}$ \\
0.18 & -0.495972 & -0.4959842 & $1.21599 * 10^{-5}$ \\
0.19 & -0.495515 & -0.495529 & $1.43025 * 10^{-5}$ \\
0.2 & -0.495033 & -0.495049 & $1.66834 * 10^{-5}$ \\
\hline
\end{tabular}

in which $v_{0}(\xi)=-\int_{0}^{\xi}\left(1 / 2 k^{2} \omega\right)(s-\xi)^{2} f_{1}\left(u_{0}(s), u_{0}^{\prime}(s), u_{0}^{\prime \prime}(s)\right.$, $\left.u_{0}^{\prime \prime \prime}(s)\right) d s$.

Then, substitute (23) into (21). We can obtain the secondorder approximate solution $u_{2}(\xi)$ :

$$
\begin{aligned}
u_{2}(\xi)= & u_{1}(\xi)+\int_{0}^{\xi} \frac{1}{2 k^{2} \omega}(s-\xi)^{2} \\
\times & {\left[k^{2} \omega u_{1}^{\prime \prime \prime}(s)-\omega u_{1}^{\prime}(s)+4 u_{1}(s) u_{1}^{\prime}(s)\right.} \\
& -3 k^{2} u_{1}(s) u_{1}^{\prime \prime}(s)-k^{2} u_{1}(s) u_{1}^{\prime \prime \prime}(s) \\
& \left.-f_{1}\left(u_{1}(s), u_{1}^{\prime}(s), u_{1}^{\prime \prime}(s), u_{1}^{\prime \prime \prime}(s)\right)\right] d s \\
= & -\frac{1}{2}+\frac{1}{2} \tanh ^{2}\left[\frac{1}{2}(x-\omega t)+\xi_{0}\right]+v_{0}(\xi) \\
+ & \int_{0}^{\xi} \frac{1}{2 k^{2} \omega}(s-\xi)^{2} \\
& \times\left[k^{2} \omega\left(u_{0}(s)+v_{0}(s)\right)^{\prime \prime \prime}\right. \\
& \quad-\omega\left(u_{0}(s)+v_{0}(s)\right)^{\prime}+4\left(u_{0}(s)+v_{0}(s)\right) \\
& \times\left(u_{0}(s)+v_{0}(s)\right)^{\prime}
\end{aligned}
$$

$$
\begin{aligned}
& -3 k^{2}\left(u_{0}(s)+v_{0}(s)\right)\left(u_{0}(s)+v_{0}(s)\right)^{\prime \prime} \\
& -k^{2}\left(u_{0}(s)+v_{0}(s)\right)\left(u_{0}(s)+v_{0}(s)\right)^{\prime \prime \prime} \\
& -f_{1}\left(u_{0}(s)+v_{0}(s), u_{0}^{\prime}(s)+v_{0}^{\prime}(s), u_{0}^{\prime \prime}(s)\right. \\
& \left.\left.+v_{0}^{\prime \prime}(s), u_{0}^{\prime \prime \prime}(s)+v_{0}^{\prime \prime \prime}(s)\right)\right] d s .
\end{aligned}
$$

Using the same method, we can get the higher order approximate solution.

\section{The Optical Soliton Perturbation Solution and the Numerical Example}

Specially, we set

$$
f=\varepsilon g(u)=\varepsilon u, \quad 0<\varepsilon \ll 1 .
$$

Then (12) change to

$$
u_{t}-u_{x x t}+4 u u_{x}-3 u u_{x x}-u u_{x x x}=\varepsilon g(u)=\varepsilon u .
$$


We obtain the zero-order and the one-order approximate solution of (20)

$$
\begin{aligned}
& u_{0}(\xi)=-\frac{1}{2}+\frac{1}{2} \tanh ^{2}\left[\frac{1}{2}(x-\omega t)+\xi_{0}\right] \\
& =-\frac{1}{2}+\frac{1}{2} \tanh ^{2}(\xi), \quad k=\frac{1}{2}, \\
& u_{1}(\xi)=u_{0}(\xi)+\int_{0}^{\xi} \frac{1}{2 k^{2} \omega}(s-\xi)^{2}(-\varepsilon u(s)) d s \\
& =-\frac{1}{2}+\frac{1}{2} \tanh ^{2} \xi \\
& -\frac{\varepsilon}{2 k^{2} \omega} \int_{0}^{\xi} s^{2}\left(-\frac{1}{2}+\frac{1}{2} \tanh ^{2} s\right) d s \\
& +\frac{\varepsilon \xi}{2 k^{2} \omega} \int_{0}^{\xi} s\left(-\frac{1}{2}+\frac{1}{2} \tanh ^{2} s\right) d s \\
& -\frac{\varepsilon \xi^{2}}{2 k^{2} \omega} \int_{0}^{\xi}\left(-\frac{1}{2}+\frac{1}{2} \tanh ^{2} s\right) d s \\
& =-\frac{1}{2}+\frac{1}{2} \tanh ^{2} \xi+\frac{\varepsilon}{2 k^{2} \omega} \int_{0}^{\xi} \ln \cosh s d s \\
& =-\frac{1}{2}+\frac{1}{2} \tanh ^{2}\left[\frac{1}{2}(x-\omega t)+\xi_{0}\right] \\
& +\frac{2 \varepsilon}{\omega} \int_{0}^{\xi} \ln \cosh s d s, \quad k=\frac{1}{2} \text {. }
\end{aligned}
$$

We set

$$
\begin{aligned}
& \xi_{0}=0, \quad \omega=1, \quad t=0, \quad \varepsilon=0.1, \\
& \xi_{0}=0, \quad \omega=1, \quad t=0, \quad \varepsilon=0.05 \text {; }
\end{aligned}
$$

we will obtain the following numerical example. See Tables 1 and 2.

\section{Conclusion}

By the analysis of structure on the left side of (8) and the properties of $f$ about the variable and the analytical variational iteration formula, we can prove that the sequence of functions of $\left\{u_{n}(\xi)\right\}$ decided by (21) is uniform convergence. So the limit function of $\left\{u_{n}(\xi)\right\}$ is the solution of the equation. Moreover, the zero-order approximate solution $u_{0}(\xi)$ is the soliton of (8) in which $f=0$; it should be specially pointed out that the more accurate the identification of the multiplier is, the faster the approximations converge to their exact solution.

\section{Conflict of Interests}

The authors declare that there is no conflict of interests regarding the publication of this paper.

\section{Acknowledgment}

The work is supported by the Youth Foundation of National Natural Science Foundation of China (Grant no. 71101072).

\section{References}

[1] M. J. McPhaden and D. Zhang, "Slowdown of the meridional overturning circulation in the upper Pacific Ocean," Nature, vol. 415, no. 6872, pp. 603-608, 2002.

[2] D. Gu and S. G. H. Philander, "Interdecadal climate fluctuations that depend on exchanges between the tropics and extratropics," Science, vol. 275, no. 5301, pp. 805-807, 1997.

[3] S. K. Liu, Z. T. Fu, and S. D. Liu, "The envelope periodic nonlinear wave equations with Jacobi Elliptic function solutions," Acta Physica Sinica, vol. 51, no. 1, pp. 10-14, 2002 (Chinese).

[4] W. Malfliet, "Solitary wave solutions of nonlinear wave equations," American Journal of Physics, vol. 60, no. 7, pp. 650-654, 1992.

[5] E. Fan and H. Zhang, "A note on the homogeneous balance method," Physics Letters A, vol. 246, no. 5, pp. 403-406, 1998.

[6] P. Rosenau and J. M. Hyman, "Compactons: solitons with finite wavelength," Physical Review Letters, vol. 70, no. 5, pp. 564-567, 1993.

[7] J. H. He, "Approximate solution of non linear differential equations with convolution product nonlinearities," Computer Methods in Applied Mechanics and Engineering, vol. 167, no. 1-2, pp. 69-73, 1998.

[8] J.-H. He, "Approximate analytical solution for seepage flow with fractional derivatives in porous media," Computer Methods in Applied Mechanics and Engineering, vol. 167, no. 1-2, pp. 57-68, 1998.

[9] J.-H. He, "Variational iteration method for autonomous ordinary differential systems," Applied Mathematics and Computation, vol. 114, no. 2-3, pp. 115-123, 2000.

[10] J.-H. He, "Variational principles for some nonlinear partial differential equations with variable coefficients," Chaos, Solitons \& Fractals, vol. 19, no. 4, pp. 847-851, 2004.

[11] J.-H. He, "Some asymptotic methods for strongly nonlinear equations," International Journal of Modern Physics B, vol. 20, no. 10, pp. 1141-1199, 2006.

[12] J.-H. He and X.-H. Wu, "Variational iteration method: new development and applications," Computers \& Mathematics with Applications, vol. 54, no. 7-8, pp. 881-894, 2007.

[13] J.-H. He, "Variational iteration method-some recent results and new interpretations," Journal of Computational and Applied Mathematics, vol. 207, no. 1, pp. 3-17, 2007.

[14] A.-M. Wazwaz, "The variational iteration method: a reliable analytic tool for solving linear and nonlinear wave equations," Computers \& Mathematics with Applications, vol. 54, no. 7-8, pp. 926-932, 2007.

[15] D. Khojasteh Salkuyeh, "Convergence of the variational iteration method for solving linear systems of ODEs with constant coefficients," Computers \& Mathematics with Applications, vol. 56, no. 8, pp. 2027-2033, 2008.

[16] S. Momani and S. Abuasad, "Application of He's variational iteration method to Helmholtz equation," Chaos, Solitons \& Fractals, vol. 27, no. 5, pp. 1119-1123, 2006.

[17] F. Geng, "A modified variational iteration method for solving Riccati differential equations," Computers \& Mathematics with Applications, vol. 60, no. 7, pp. 1868-1872, 2010. 
[18] S. Das, "Solution of fractional vibration equation by the variational iteration method and modified decomposition method," International Journal of Nonlinear Sciences and Numerical Simulation, vol. 9, no. 4, pp. 361-366, 2008.

[19] L. Xu, "The variational iteration method for fourth order boundary value problems," Chaos, Solitons and Fractals, vol. 39, no. 3, pp. 1386-1394, 2009.

[20] M. A. Noor and S. T. Mohyud-Din, "Variational iteration method for solving higher-order nonlinear boundary value problems using He's polynomials," International Journal of Nonlinear Sciences and Numerical Simulation, vol. 9, no. 2, pp. 141-156, 2007.

[21] M. A. Noor, K. I. Noor, and S. T. Mohyud-Din, "Modified variational iteration technique for solving singular fourth-order parabolic partial differential equations," Nonlinear Analysis: Theory, Methods \& Applications, vol. 71, no. 12, pp. e630-e640, 2009.

[22] A. Ghorbani and J. Saberi-Nadjafi, "An effective modification of He's variational iteration method," Nonlinear Analysis: Real World Applications, vol. 10, no. 5, pp. 2828-2833, 2009.

[23] A. Sevimlican, “Constructing approximate Green's function for a vector equation for the electric field using the variational iteration method," Applied Mathematics Letters, vol. 23, no. 5, pp. 533-536, 2010.

[24] M. Inokuti, H. Sekine, and T. Mura, "General use of the Lagrange multiplier in nonlinear mathematical physics," in Variational Method in the Mechanics of Solids, S. Nemat-Nasser, Ed., pp. 159-162, Pergamon Press, NewYork, NY, USA, 1978.

[25] J.-H. He, "Variational iteration method-a kind of non-linear analytical technique: some examples," International Journal of Non-Linear Mechanics, vol. 34, no. 4, pp. 699-708, 1999.

[26] A.-M. Wazwaz, "A comparison between the variational iteration method and Adomian decomposition method," Journal of Computational and Applied Mathematics, vol. 207, no. 1, pp. 129136, 2007.

[27] T. A. Abassy, M. A. El-Tawil, and H. El Zoheiry, "Solving nonlinear partial differential equations using the modified variational iteration Padé technique," Journal of Computational and Applied Mathematics, vol. 207, no. 1, pp. 73-91, 2007.

[28] Z. M. Odibat, "Reliable approaches of variational iteration method for nonlinear operators," Mathematical and Computer Modelling, vol. 48, no. 1-2, pp. 222-231, 2008.

[29] M. Tatari and M. Dehghan, "Improvement of He's variational iteration method for solving systems of differential equations," Computers \& Mathematics with Applications, vol. 58, no. 11-12, pp. 2160-2166, 2009.

[30] Z. M. Odibat, "A study on the convergence of variational iteration method," Mathematical and Computer Modelling, vol. 51, no. 9-10, pp. 1181-1192, 2010.

[31] A. Degasperis and M. Procesi, Asymoptotic Integrability, Symmetry and Perturbation Theory, World Scientific, River Edge, NJ, USA, 1999.

[32] A. A. Soliman, "A numerical simulation and explicit solutions of KdV-Burgers' and Lax's seventh-order KdV equations," Chaos, Solitons and Fractals, vol. 29, no. 2, pp. 294-302, 2006.

[33] R. Camassa and D. D. Holm, "An integrable shallow water equation with peaked solitons," Physical Review Letters, vol. 71, no. 11, pp. 1661-1664, 1993.

[34] L. Q. Yu and L. X. Tian, "A new traveling wave solutions for the Degasperis-Procesi equation," Journal of Jiangsu University, vol. 26, no. 3, pp. 231-234, 2005 (Chinese).
[35] J. H. He, Approximate Nonlinear Analytical Methods in Engineering and Sciences, Henan Science and Technology Press, Zhengzhou, China, 2002, (Chinese). 


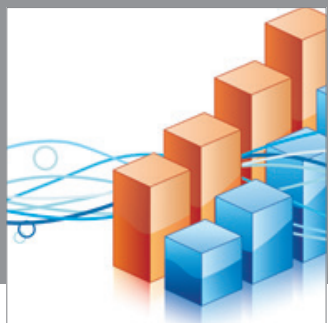

Advances in

Operations Research

mansans

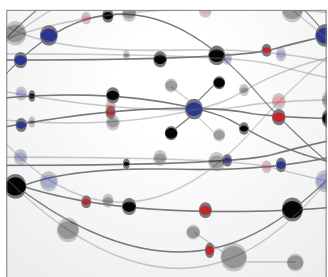

The Scientific World Journal
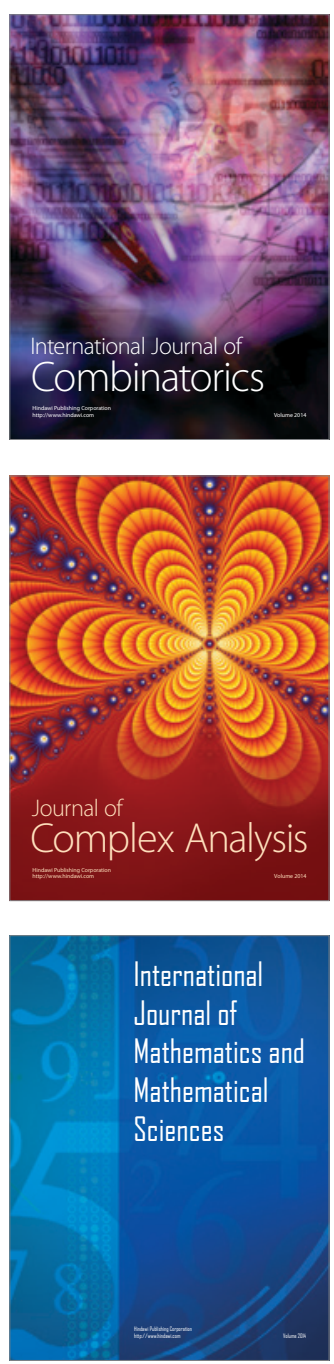
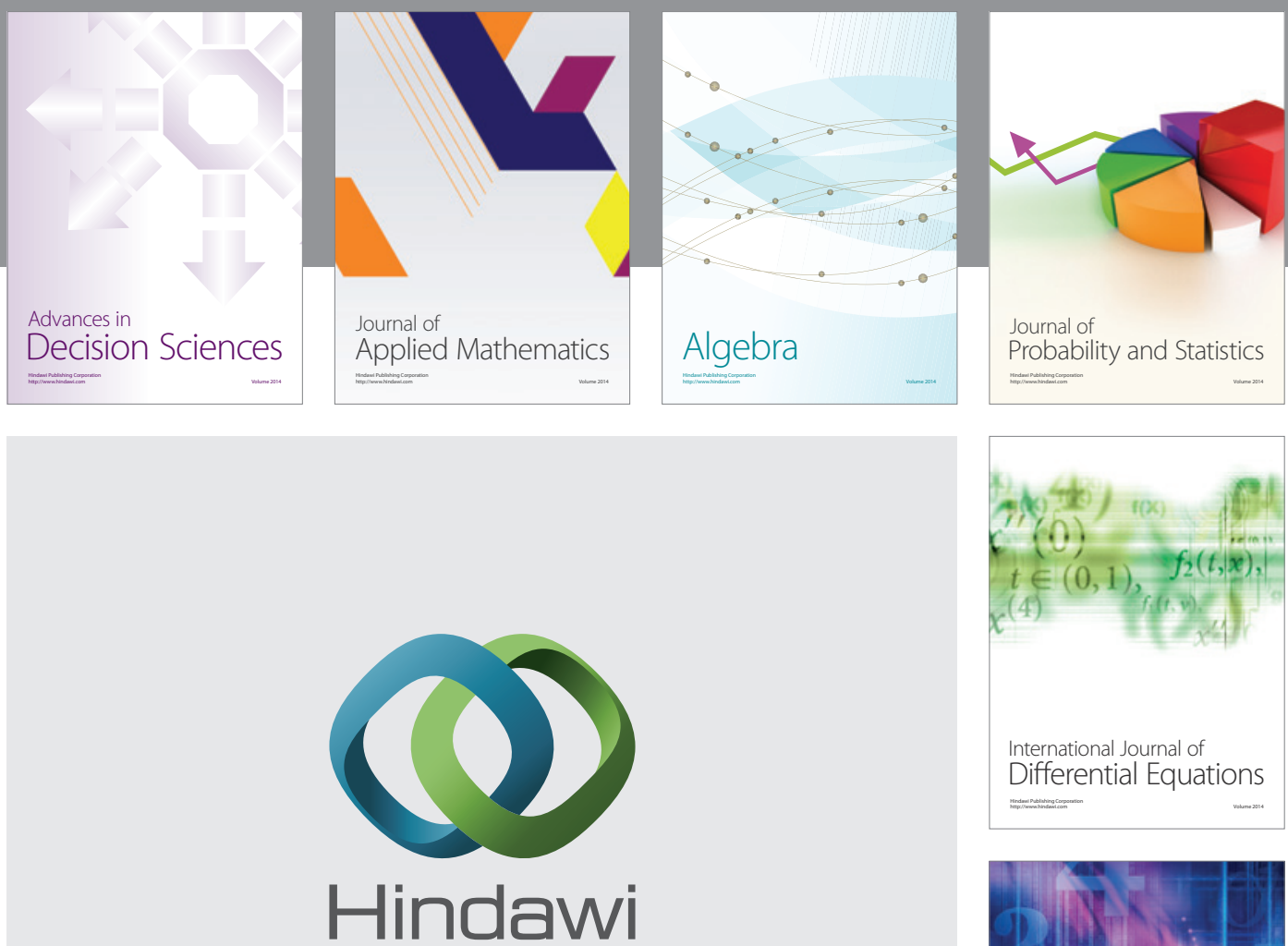

Submit your manuscripts at http://www.hindawi.com
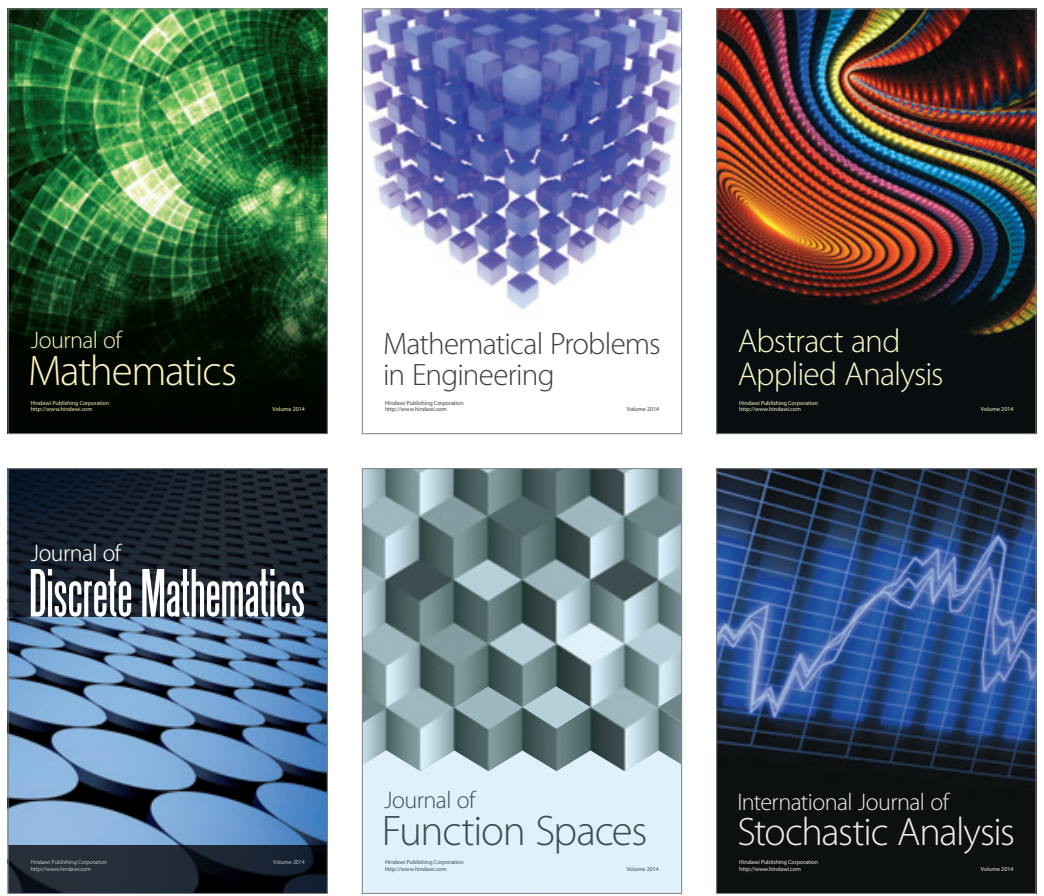

Journal of

Function Spaces

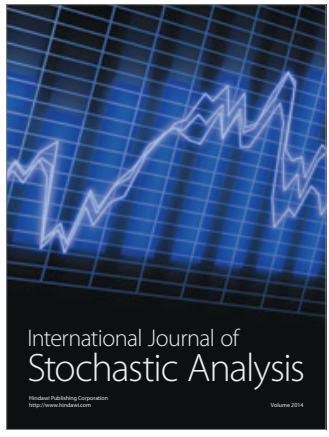

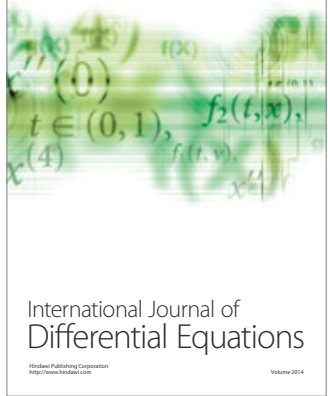
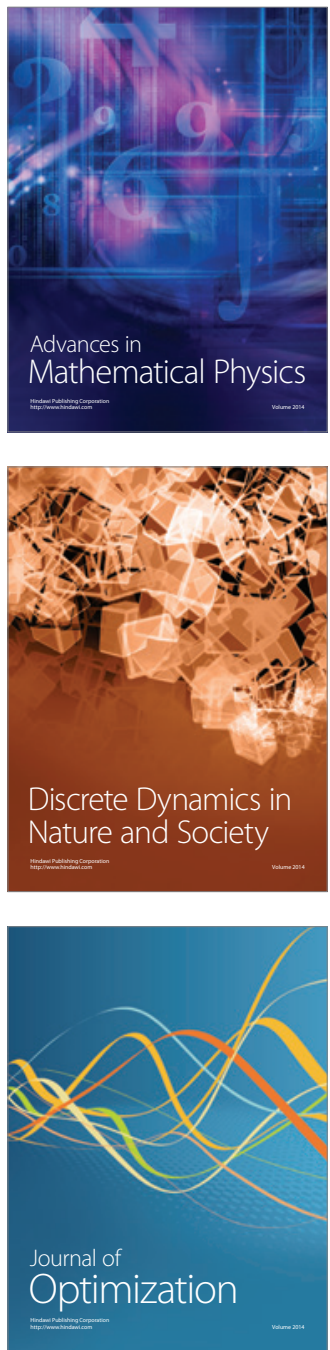